the Hughes Corporation ${ }^{24}$ ) had a sensitivity of roughly $1 \cdot 7 \cdot 10^{-13} \mathrm{~cm} / \mathrm{VHz}$ at $\sim 5 \mathrm{kHz}$ for an interferometer 850 $\mathrm{cm}$ long, which is equivalent to about $10^{3} \mathrm{GPU}$.

It is nevertheless possible that a network of very sensitive laser interferometers operating on base lines of many $\mathrm{km}$ be put into operation in space near the Earth in the distant future, telling us about all changes of the space time geometry which occur in the Universe.

\section{REFERENCES}

1. LEE, M, GRETZ, D, STEPPEL, $S$, and WEBER, J. "Gravitational Radiation Detector Observations in 1973 and 1974" Phys. Rev. D 19 (1976) 893.

2. BRAGINSKII, V.B., MANUKIN, A.B., POGOV, E.I., RUDENKO, V.N. and KHORER, A.A. "An Upper Limit on the Density of Gravitational Radiation of Extraterrestrial Origin" Sov. Phys. JETP 39 (1974) 387

3. LEVINE, J.L. and GARWIN, B.L. "New Negative Result for Gravitational Wave Detection and Comparison with Reported Detector" Phys. Rev. Lett. 33, (1974) 794. 4. DOUGLASS, D.H., GRAM, R.Q., TYSON, J.A. and LEE R.W. "Two Detector Coincidence Search for Bursts of Gravitational Radiation", Phys. Rev. Lett. 35 (1975) 480.

5. BILLING, H., KAFKA, P., MAISCHBERGER, K., MEYER, F. and WINKLER, W.

"Results on the Munich-Frascati Gravi- tational Wave Experiment" Lett. Nuovo Cimento 12 (1975) 111

6. BILLING, $H$. and WINKLER, W. "The Münich Gravitational Wave Detector" Nuovo Cimento 33B (1976) 665.

7. KAFKA, P. Proc. of the International School of Cosmology and Gravitation, Erice 1975, J. Weber ed. (Plenum Press, New York) 1977.

8. BONNAZZOLA, S. CHEVRETON, M., FELENBOK, P., HERPE, G. and THIERRYMIEG, J. "Meudon Gravitational Radiation Detection Experiment" Colloques Internationaux C.N.R.S. N. 220, June 1973 (Paris).

9. DREVER, B.W.P., HOUGH, J., BLAND, B. and LESSNOFF G.W. "Search for Short Bursts of Gravitational Radiation" Nature 246 (1973) 340.

10. APLIN, P.S. "An Improved Detector of Gravitational Radiation" GRG 3111 (1972). 11. ALLEN, W.D. and CHRISTODOULIDES, C. "Gravitational Radiation Experiments at the University of Reading and the Rutherford laboratory" J. Phys. A. Math. Gen. 8 (1975) 1726

12. HIRAKAWA, $H$. and NARIHARA, K. "Search for Gravitational Radiation at 145 Hz" Phys. Rev. Lett. 35 (1975) 330. 13. MAEDER, D.G. and SANDERSON, B., "Sensitivity Calibration and Electronic Simulation of Gravitational Burst Detectors" J. Phys. E Sci. Instr. 8 (1975) 920. 14 PIZZELLA, G., Report at the 4th Soviet gravitational Conference, Minsk, June 1976.

15. AMALDI, E. et al. "The Cryogenic Gravitational Wave Experiment in Rome: Progress Report, Sept. 1977" Nota Interna n. 697,18 novembre 1977, Istituto di Fisica, Roma.

16. BOUGHN, S.P. et al. "Observation of
Mechanical Nyquist Noise in a Cryogenic Gravitational Wave Antenna" Phys. Rev. Lett. 38 (1977) 454.

17. DAVIS, W., GRETZ, D., RICHARD, J.P. and WEBER, J., "Development of Cryogenic Gravitational Wave Antennas at the University of Maryland" Report at GR8, Waterloo, Aug. 1977.

18. OELFKE, W.C. and HAMILTON, W.O., "Superconducting Accelerometers for the Study of Gravitation and Gravitational Radiation" XXVII Congress, IAF, California, October 1976.

19. RAND, R.E, and BLAIR, D.G "The Gravitational Wave Project at the University of Western Australia" Australian Physicist 14 (1977) 132 and 151.

20. BRAGINSKII, V. B. and VORONTSOV, Yu. I. Usp. Fiz Nauk 114, 41 (1974) (Sov. Phys.-Usp. 17 644, 1975).

21. THORNE, K.S. et al. "Quantum Nondemolition Measurements of Harmonic Oscillators" Submitted to Phys. Rev. Letters (1978).

22. BARTON, R. J., PAPINI, G., RAMADAM, B., STRAYER, D. and TWARD, E. "Cryogenic Gravitational Wave Detector using Single Crystals" Proc. Symposium on Experimental Gravitation, Pavia, September 1976.

23. WEISS, R. Quarterly Progress Report, Research Lab. of Electronics MIT 105, 54 (1972).

24. WINKLER, W. "A Laser Interferometer to Search for Gravitational Waves" Proc. Symposium on Experimental Gravitation, Pavia, September 1976.

25. FORWARD, R.L., and MOSS, G.E. "Wideband Interferometric Gravitational Radiation Antenna" Meeting American Physical Soc., Los Angeles, Cal. December 1972.

\title{
Neutron Scattering Experiments and New Theoretical Approaches
}

The 7th Europhysics Conference on Macromolecular Physics was held in Strasbourg from May 23 to 26 . Despite the crowded calendar of meetings on polymer science, the EPS conferences have assumed an important role as they are dealing, on a European level, with well defined topics of current interest. The self-imposed restriction in the scope of the conference theme as well as the active international participation are advantages which permit a highly intensive coverage. At the same time they circumvent the problems of many large international conferences (numerous and short presentations in parallel sessions) or of national meetings (limited number of contributions in a particular area).

Following the first meeting of the Macromolecular Physics Section in 1973 , these topical EPS conferences have come to be considered by experts in the specialized fields as a forum for intensive discussions and for an exchange of latest results. The meetings are also attended, however, by the general polymer physicist who merely wishes to obtain refined information on e.g. structure or mobility. Europhysics Conference Abstracts are also increasingly used as a suitable, fast, and up-to-date reference to recent European progress in a particular field.

The above rather general remarks have been made as they both characterize the intentions of the Section and apply to the Conference in Strasbourg. There, 115 participants followed 8 main lectures and 32 contributed papers. Whereas these figures are quite typical for the size and programme organization of the EPS Polymer meetings it is regretted that there was no participation by eastern European countries, possibly due to the fact that neutron scattering activities in these countries are on a relatively low level.
In his report on quasi-elastic and inelastic scattering J. Higgins (London) reviewed the information on molecular motion to be obtained from the variation of coherent and incoherent scattering cross-sections with neutron wave vector $\pi$. She stated that in bulk rubbery samples, well above their glass transition temperatures, a fast segmental motion had been observed. Although the motion is somewhat slower than that in dilute solution, it is many orders of magnitude faster than translational diffusion of the whole molecule. In that case the Rouse model of normal modes for a "bead-spring" chain gave a surprisingly good fit to the data and the full energy width of the observed scattering law varies as $x^{n}$ with $3<n<4$ in most cases. The value of $n$ increased with temperature and with chain flexibility with an apparent upper limit of 4. Her lecture gave an excellent introduction into this area. Using this presentation as an example 
it is suggested that the impact of the Conference Abstracts would be strengthened by providing more space to one (or more) of the invited lecturers for a complete version of their highly informative papers. In any event the abstracts of invited lectures should contain the leading references.

Kirste (Mainz) and Schelten (Jülich), who were among the first to apply neutron scattering to an elucidation of the near order structure of polymers, talked about their subjects, Kirste treating amorphous materials, particularly the beginning of phase separation in blends of polystyrene-co-acrylonitriles (PSAN) of different AN content and Schelten problems of the "adjacent re-entry" in semicrystalline polymers (experimental errors of scattering data, nitric acid etching to confirm escape of molecules from a crystallite, comparison with Flory-Yoon calculations). The presentation of Schelten and later talks from Mainz and Bristol have shown that three groups presently working on the conformation of chains in semicrystalline polymers are now approaching identical conclusions; that is, essentially re-entrant chain folding in single crystals grown from solution, and no regular folding in the rapidly crystallized melt. This agreement seems to be one of the main achievements of the meeting owing to the rather controversial nature of this topic.

Then came Carpenter (Chicago) who reported on new instrumental developments. Pulsed neutron sources used with large area detectors widen the range of wave lengths $\lambda$ available which are sorted by time-of-flight diffractometers with $\Delta \lambda / \lambda<0.01$.
Contributed papers dealt, for example, with the conformation of $n$-alkane molecules in the melt and in solution, with local orientation correlation in partially crystallized polymers (also studied by the anisotropy of thermal diffusivity), with "reptational motions" of diffusing molecules (corroborated by micro-IR measurements), and with the conformation of chains in drawn polystyrene (using also partially labelled chains). The latter experiments confirmed an affine deformation of the average chain end-to-end vectors but revealed a degree of orientation for the molecular segments, which deviated the more from an affine deformation the higher the arawing temperature.

H.H. Kausch (EPFL, Lausanne)

\section{- ECOSS I - Europe Comes Together on Surface Science}

Surface Science is not only dynamic and rapidly developing, but might even be considered to be growing mature ; so mature in fact, that it is now appropriate to replace the several biennial, quasi-international conferences by a joint annual European Conference on Surface Science. The first, organized under the auspices of EPS, IUPAP, and IUVSTA, was held in Amsterdam, 5-9 June, 1978.

Progress as reported at this meeting is, indeed, taking place on a very wide front. This reflects the breadth of the field, which could be defined as either "multidisciplinary, interdisciplinary or as a sub-discipline on its own " 1 ). The Surface attracts physicists, chemists, and technologists, and within physics alone, contributions are made by people with a great variety of background - from atomic, molecular, and solid-state physics. This makes the picking-out of conference highlights difficult and a matter of personal interest.

The Conference gave me the definite impression that we have now gone a long way towards experimentally characterizing the atomic and electronic structures of surfaces, and that it is becoming more and more proper to ask questions about how to use the increasingly detailed information thus obtained. This leads us to the core of Surface Science ; issues that earlier were fashionable essentially as research proposals (corrosion, heterogeneous catalysis, ...) are today, subjects for active surface-science research.

Judging from the Conference $\mathrm{Ab}$ stracts ${ }^{2}$ ), structure, electron properties, chemisorption and catalysis should be the major fields of interest right now. Taking this in conjunction with the impression gained at last year's international conference in Vienna, one sees a clear trend of methods becoming established for the determination of atomic and electronic structure of surfaces and a strongly growing interest in surface reactions.

The Nestor among the structuredetermining methods, low-energyelectron diffraction (LEED) by now has revealed almost 100 surface structures, and one now dares to talk about surface crystallography as a discipline. Such a term would denote a wide area and include many other techniques. For instance, new medium energy-ion-scattering data were reported, which resolve the position of a sulphur atom on a nickel surface with a claimed accuracy of $0.01 \AA$. Methods utilizing $X$-rays for excitation and diffracted Auger- or photoelectrons for detection seem to have got off the ground, and angularresolved photoemission in the ultraviolet and electron-energy-loss spectroscopy are also giving valuable supplementary information about, in particular, sites of adsorbed species. The overwhelming majority of the structural data is still nevertheless coming from LEED, with a claimed accuracy of about 0.05-0.01 § perpendicular, and $0.2 \AA$ parallel to the surface. The great progress made during the past years now makes it possible to obtain a coherent picture of, e.g., certain adsorbate-substrate combinations.

The study of the electron structure is dominated by photoelectron spectroscopy. Angular resolution, in particular when used with polarized synchrotron radiation in the ultraviolet, permits the direct determination of the dispersion curves for surface and bulk states, as well as of band/ adsorbate orbital symmetries. For chemisorption and surface reactions it gives information about ionization potentials and provides "finger-prints" of adsorbed species. Ultraviolet photoemission can now give electron energies with an accuracy that should be provoking for the theorists.

Another problem that occupies several theorists, concerns shake-up and relaxation processes occuring upon photoexcitation of core electrons in adsorbed species. There is now some understanding of the additional structures that appear in the adsorbate core level photoelectron spectra and which are not present in the corresponding spectra of atoms and molecules in the gas phase.

The long list of surface-reaction papers clearly indicates that progress has been made at a rapid rate in the fields of oxidation, adsorption of molecules - not just the favourite proto- 